\title{
Demanda residencial por energia elétrica no Brasil (2004-2015)
}

\author{
Víctor Henriques de Oliveira* \\ Ricardo de Souza Tavares** \\ Luís Antônio Tavares ***
}

\begin{abstract}
Resumo
O fornecimento de energia elétrica é um serviço indispensável para a economia. É uma condição básica para o desenvolvimento das atividades cotidianas dos agentes, tendo implicações sobre nível de bem-estar de uma sociedade. Sob esta perspectiva, este trabalho tem como objetivo estimar o impacto de variações de renda e preço sobre a demanda residencial por energia elétrica no Brasil durante o período 2004-2015. Para tanto, foram utilizados Vetores Autorregressivos (VAR) e testes de Cointegração para obter as elasticidades preço e renda. Os resultados evidenciam que, em média, os consumidores reagem positivamente a elevações de renda, enquanto variações positivas na tarifa do serviço de eletricidade e nos preços dos elétrodomésticos impactam negativamente o consumo residencial de eletricidade. Ademais, as estimativas sugerem uma demanda muito inelástica no Brasil. Alterações na renda e nos preços exercem um pequeno impacto sobre a quantidade consumida de energia elétrica. Estes resultados estão de acordo com estudos anteriores, o que revela a necessidade do desenvolvimento de políticas setoriais. Órgãos de regulação devem estabelecer políticas tarifárias ótimas, com o objetivo de oferecer um serviço mais eficiente e acessível aos cidadãos brasileiros.
\end{abstract}

Palavras-chave: demanda residencial; vetores autoregressivos; cointegração.

\section{Residential demand for electricity in Brazil (2004-2015)}

\begin{abstract}
The supply of electricity is a highly important service in the economy. It is a basic condition for agent's daily activities, which has implications on society level of welfare. From this perspective, this paper aims to estimate the impact of changes in income and prices on household demand for electricity in Brazil during 2004-2015. We use Vectors Autoregressive (VAR) and Cointegration tests to obtain prices and income elasticities. The results shows that, on average, consumers react positively to income increases, while positive changes in electricity service tariffs and electrical equipment prices have a negative impact on residential electricity consumption. Besides, the outcome suggests a highly inelastic demand in Brazil. Both changes in income and prices have low impact on the amount of electricity consumption. It has already been described in Brazilian publications, revealing the need for sectorial policies. Regulatory agencies should work on establishing optimal tariff policies, aiming a more efficient and accessible service to Brazilian citizens.
\end{abstract}

Keywords: household demand; vectors autoregressive; cointegration.

JEL: Q4, Q41

\footnotetext{
* Mestrando em Economia Aplicada pelo Programa de Pós-Graduação em Economia da Universidade Federal do Rio Grande do Sul (UFRGS). E-mail: victorhenriquesoliv@gmail.com

** Mestrando em Economia Aplicada pelo Programa de Pós-Graduação em Economia da Universidade Federal do Rio Grande do Sul (UFRGS). E-mail: UFRGS. - s17tavaresricardo@gmail.com

***Professor do Instituto Federal de Educação, Ciência e Tecnologia do Sul de Minas (IFSULDEMINAS), Campus Pouso Alegre - MG. E-mail: luis.tavares@ifsuldeminas.edu.br
} 
Revista Catarinense de Economia - RCE

APEC - Associação dos Pesquisadores em Economia Catarinense

$1^{\circ}$ Semestre de 2018 - www.apec.pro.br/rce

\section{Introdução}

Como definido pelo Operador Nacional do Sistema Elétrico (ONS), o Sistema Elétrico Brasileiro (SEB) é majoritariamente hidrotérmico ${ }^{1}$ de grande porte e pode ser caracterizado como único, em âmbito mundial, devido às suas características, tais como sua extensão e presença de vários proprietários atuantes no setor.

O SEB passou por profundas transformações econômicas e estruturais nas últimas duas décadas. O governo brasileiro controlava quase todo o setor de energia elétrica no país, tanto na esfera federal como na estadual. No entanto, em 1995, teve início seu processo de privatização através do Programa Nacional de Desestatização (PND), com a finalidade de dinamizar o setor e melhorar a qualidade do serviço ofertado. De acordo com Silva (2011), a Lei Geral das Concessões abriu precedentes para uma restruturação do sistema, dando espaço a um novo modelo estrutural, o chamado RE-SEB. Implantado em 1996, este possibilitou a desverticalização da cadeia produtiva em linhas de transmissão, geração, distribuição e comercialização de energia elétrica. Estas mudanças tornaram o setor mais articulado e competitivo ao promover a interação entre diversos agentes em diferentes mercados sobre uma mesma região, o que, por sua vez, possibilitou o fim das reservas de mercado no setor.

Contudo, como ressalta Sales (2002), problemas de coordenação entre os órgãos reguladores e as falhas no processo de transição do setor público para o privado emperraram o processo de desenvolvimento do setor. Além disso, o primeiro biênio do século XXI foi marcado pela grave crise de oferta energética devido à forte crise hídrica que assolava o Brasil. Com isso, o governo propôs o racionamento de até $20 \%$ da energia elétrica consumida pela classe residencial.

Diante destes episódios, criou-se a necessidade de transformar efetivamente o setor elétrico. Nesse sentido, Silva (2011) destaca que a restruturação do novo modelo do SEB, no final de 2004, contou com a criação da Empresa de Pesquisa Energética (EPE) e da Câmara de Comercialização de Energia Elétrica (CCEE). A primeira objetivava o desenvolvimento de planos setoriais e estudos do mercado energético, enquanto a segunda assegurava a estabilidade da oferta de energia elétrica ao possibilitar a sua contratação eficiente.

Desde então, observou-se que a demanda residencial por energia elétrica cresceu consideravelmente. O consumo residencial de energia elétrica na rede básica cresceu 4,7\% na

\footnotetext{
${ }^{1} \mathrm{O}$ termo hidrotérmico se refere ao sistema composto pela geração de energia hidroelétrica e termoelétricas, interligadas por um sistema de rede de transmissão que conecta as usinas aos centros de consumo. (ONS, 2011).
} 
Revista Catarinense de Economia - RCE

APEC - Associação dos Pesquisadores em Economia Catarinense

$1^{\circ}$ Semestre de 2018 - www.apec.pro.br/rce

última década, isto é, passou de 82.644 gigawatts-hora consumidos, em 2005, para 131.315 gigawatts-hora no final do ano de 2015 (EPE, 2016).

Contudo, de acordo com Monteiro (2015), os efeitos do realinhamento dos preços de energia elétrica, junto com a revisão tarifária extraordinária (RTE) elevaram o preço da tarifa média em 36,34\% no ano de 2015, provocando alterações significativas no consumo residencial em 2015.

De fato, o consumo de energia elétrica tende a maximizar a utilidade geral de uma sociedade. Em outras palavras, o consumidor obtém maior satisfação à medida em que o serviço de eletricidade é usufruído. Seja pela assistência nas atividades cotidianas, pela necessidade básica da iluminação ou até mesmo para seu conforto e lazer, o consumo de energia proporciona a melhoria da qualidade de vida dos indivíduos. Portanto, alterações na renda e/ou nos preços podem mudar o seu padrão de consumo e, em alguns casos, privá-los de atender todas às suas necessidades, reduzindo seu bem-estar, especialmente no caso das famílias mais carentes.

Desta maneira, diversos economistas passaram a estudar a relação das variáveis econômicas e o consumo de eletricidade. Em linhas gerais, a demanda residencial por energia elétrica tem sido analisada através da estimação das elasticidades, com a finalidade de mensurar a sensibilidade do consumo perante as variações na renda disponível dos indivíduos e dos preços das tarifas médias.

Os estudos sobre a demanda residencial por energia elétrica publicados remontam discussões acerca do bem-estar dos consumidores, da eficiência do consumo, da preocupação com o fornecimento de energia e dos impactos envolvendo o meio-ambiente. Em particular, procurou-se esclarecer a relação consumo de energia com a renda e os preços.

Houthakker (1951), um dos trabalhos precursores sobre demanda de energia elétrica, investigou a demanda residencial de energia elétrica para 42 cidades da Grã-Bretanha entre 1937 e 1938. No que tange o preço do serviço de energia elétrica, o autor adotou preços marginais em vez de preços médios para a estimativa da tarifa. Os resultados do estudo evidenciam o caráter elástico, em relação à renda, e pouco elástico para preço da tarifa de energia.

Bentzen e Engsted (1993) estimaram a demanda residencial por eletricidade da Dinamarca no período 1948-1990. O resultado apresentado para a elasticidade-renda sugere que impactos na renda teriam um efeito maior do que a unidade no longo prazo, evidenciando seu caráter elástico em relação ao consumo residencial de energia elétrica. Em contrapartida, a elasticidade-preço de longo prazo foi considerada inelástica em relação ao consumo de 
Revista Catarinense de Economia - RCE

APEC - Associação dos Pesquisadores em Economia Catarinense

$1^{\circ}$ Semestre de 2018 - www.apec.pro.br/rce

eletricidade, indicando que alterações positivas no preço das tarifas provocariam diminuições menos do que proporcionais no consumo, neste caso, inferiores à unidade.

Diferentemente dos dois trabalhos mencionados sobre a elasticidade da demanda de energia elétrica, Silk e Joutz (1997) incluíram os preços de fontes alternativas de energia como de combustíveis fósseis e também incorporaram os efeitos de temperaturas dos dias mais quentes e mais frios no modelo estimado para o período 1949-1993.

Na literatura brasileira, Modiano (1984) foi o pioneiro em trabalhos sobre a demanda de energia elétrica. $\mathrm{O}$ autor estimou a demanda por energia elétrica, a partir de dados anuais compreendidos entre 1961-1981 para as quatro classes de consumo: residencial, industrial, comercial e outras. No entanto, admite que sua estimativa esteja viesada, dada a relação de simultaneidade na determinação dos preços e do consumo de energia elétrica.

Com objetivo de atualizar e aperfeiçoar o trabalho de Modiano (1984), Andrade e Lobão (1997) analisaram somente as elasticidades da classe residencial no período 1963-1995. Foram incorporados ao modelo os preços de equipamentos elétricos, dada a considerável influência para o consumo desta classe. A estimação do modelo apresentou resultados consistentes para as elasticidade-renda, preço da tarifa e dos eletrodomésticos. Assim, os autores concluíram que a queda dos preços reais da tarifa e dos eletrodomésticos tiveram um impacto significativo no crescimento do consumo residencial de energia, dado que a duplicação da renda per capita no período possibilitou a expansão do estoque de equipamentos nos domicílios.

Um estudo mais contemporâneo no Brasil, que pode ser destacado, é o de Schmidt e Lima (2005). Com foco na análise das elasticidades de longo prazo das três principais classes de consumo (residencial, comercial e industrial), o trabalho utilizou dados anuais de 1969 até 1999. Observou-se, neste estudo, que os consumidores reagem mais às variações de preço dos eletrodomésticos do que propriamente variações no preço da tarifa média em reais por megawatt-hora.

Diferentemente dos outros autores, Gomes (2010) usou uma abordagem de dados em painel para a demanda residencial de energia elétrica do Brasil no período 1999-2006. Destacase neste trabalho, a nova metodologia empregada para estimação e a adoção das tarifas homologadas, sendo uma proxy mais apropriada para representar o preço de energia elétrica, dado que sua fixação exógena é feita pela ANEEL, o que evita problemas com endogeneidade no modelo.

O trabalho mais recente sobre a demanda residencial de energia no Brasil é o de Schutze (2015). Com aplicação de dados em painel, a autora investigou o efeito do preço da tarifa sobre o consumo residencial do Brasil, através de dados mensais de uma base agregada de 61 
Revista Catarinense de Economia - RCE

APEC - Associação dos Pesquisadores em Economia Catarinense

$1^{\circ}$ Semestre de 2018 - www.apec.pro.br/rce

concessionárias de energia elétrica no período 2005-2013. A estimativa da elasticidade-preço indica que políticas baseadas no preço desencorajam o consumo residencial, reduzindo incentivos para expansão da capacidade de geração.

Cabe ressaltar que, independente da orígem do estudo, do período escolhido e do método utilizado, os resultados empíricos desta breve revisão de literatura nacional e internacional da elasticidade-preço de longo prazo indicam que as variações no preço da tarifa implicam em variações menos do que proporcional no consumo. Estes resultados parecem refletir aspectos inerentes do serviço de energia elétrica, tais como sua essencialidade e falta de bons substitutos.

No que se refere à elasticidade-renda de longo prazo, os resultados verificados são bastante heterogêneos, sendo possível observar valores superiores e inferiores a unidade. Silk e Joutz (1997) apontam que a omissão das estimativas dos estoques de equipamentos elétricos implica na superestimação da elasticidade-renda. De forma similar, elasticidade-renda se mostrou superior a unidade naqueles trabalhos onde se omitiu o efeito dos equipamentos elétricos, como é o caso de Bentzen e Engsted (1993) e Modiano (1984), enquanto os demais trabalhos que incorporaram o componente estoque de aparelhos no modelo verificaram elasticidade-renda menor do que a unidade. É valido salientar que estes resultados foram verificados somente para os trabalhos que utilizaram séries temporais, não sendo possível afirmar o mesmo para estudos em painel.

Percebe-se também que, ao longo do tempo, os estudos nacionais têm sido analisados por meio de séries temporais, mais precisamente pelos modelos VAR e VEC, juntamente com o teste de cointegração, com a finalidade de obter estimativas mais robustas para as elasticidades da demanda residencial por energia elétrica. Entretanto, estudos com dados em painel sobre o tema ainda são incipientes no caso brasileiro. $\mathrm{O}$ que parece ter sido determinante para a adoção do VAR e do teste de cointegração na maioria dos estudos foi a captação de relações em longo prazo e pelo controle da endogeneidade da tarifa média de energia elétrica.

Em vista das recentes mudanças da conjuntura econômica do país, este estudo complementa a literatura existente sobre a demanda residencial por energia elétrica ao incorporar informações mais atuais, com o propósito de explicitar uma nova relação do consumo e de suas variáveis, tornando-o mais contemporâneo em relação aos demais. Além disso, um diferencial deste trabalho é a estimação do modelo VAR com utilização de dados mensais, já que não há casos de estudos recentes sobre o tema com esta metodologia.

Ademais, o conhecimento do comportamento do consumidor sobre as variações dos componentes da demanda residencial deste estudo para o presente momento fornece informações relevantes para que os órgãos regulatórios estabeleçam as diretrizes de políticas 
setoriais destinadas à regulação do mercado de energia elétrica, como também para o governo, ao implementar políticas públicas destinadas a aumentar o bem-estar dos consumidores residenciais.

\section{Referencial teórico}

A demanda individual por energia elétrica é tida como parte da teoria do consumidor, pois se baseia nos princípios marginalistas de maximização da utilidade e da minimização dos custos.

De acordo com a teoria neoclássica, a necessidade de satisfação própria (self-interest) é inerente ao ser humano, fato que norteia a tomada de decisão individual dos consumidores. Esta é dependente dos recursos escassos e dos preços relativos dos bens e serviços disponíveis em uma sociedade.

Dessa maneira, os indivíduos escolhem uma cesta de bens ou serviços que maximizem sua satisfação. A preferência por cada mercadoria demonstra como um consumidor individual se comporta perante a escolha de duas cestas quaisquer. Contudo, os consumidores estão sujeitos a uma dada restrição orçamentária. Tudo o mais permanecendo constante, alterações na renda provocam o deslocamento desta restrição, o que implica no alcance de uma nova curva de utilidade, que por sua vez oferece maior satisfação ao consumidor ou vice-versa.

Assim, em uma economia de mercado, o trade-off enfrentado por cada consumidor e o nível de consumo que cada qual deseja alcançar são representados pela teoria do consumidor. Logo, a demanda de um bem qualquer pode ser representada pela condição otimização:

$$
\text { Maximizar } U\left(X_{i}\right)
$$

Onde:

$$
\text { Sujeito a: } P_{i} Q_{i}+P_{j} Q_{j} \leq Y
$$

$P_{i}$ é o preço de um bem i qualquer;

$P_{j}$ é o preço de todos os outros bens consumidos;

$Q_{i}$ é a quantidade consumida do bem $i$;

$Q_{j}$ é a quantidade de todos os outros bens consumidos;

$Y$ é a renda do consumidor.

A função que determina a quantidade demandada de um bem é dada pela resolução do problema acima e pode ser representado pela expressão: 
Revista Catarinense de Economia - RCE

APEC - Associação dos Pesquisadores em Economia Catarinense

$1^{\circ}$ Semestre de 2018 - www.apec.pro.br/rce

$$
Q_{i}=f\left(Y, P_{i}, P_{j}\right)
$$

Em linhas gerais, a quantidade demandada de energia elétrica depende dos mesmos fatores econômicos observados na expressão (1). Assim, a quantidade de eletricidade que o indivíduo deseja consumir depende da tarifa cobrada por este serviço, mais o estoque de equipamentos que via de regra necessita de eletricidade para seu uso e do modo como ele aloca o seu rendimento entre estes componentes. Algebricamente, esta relação pode ser expressa por:

$$
Q_{t}=f\left(Y_{t}, T_{t} E_{t}\right)
$$

Em que:

$Q_{t}$ é a quantidade consumida de energia elétrica:

$Y_{t}$ é a renda do consumidor:

$T_{t}$ é a tarifa cobrada pelo serviço:

$E_{t}$ é o estoque de equipamentos elétricos.

Por hipótese, a energia elétrica é vista como um bem necessário, portanto, um aumento da renda pode elevar o consumo de eletricidade das famílias. $\mathrm{O}$ aumento da renda também pode influenciar o consumidor a comprar mais equipamentos elétricos, ampliando o seu estoque de equipamentos, o que também ocasionaria em uma expansão do consumo de energia elétrica (MATTOS, 2005).

Em relação à tarifa cobrada pelo serviço de eletricidade, os estudos nacionais em quase sua totalidade têm usado as tarifas médias como proxy do preço de energia elétrica. Novamente, Mattos (2005) destaca que as tarifas médias são de conhecimento público e os consumidores residenciais adaptam o seu respectivo consumo de acordo com o preço médio praticado, tornando-se uma boa proxy para responder às alterações na quantidade demandada de energia elétrica.

Nessa perspectiva, o que se pretende analisar é a sensibilidade da quantidade demandada perante às variações dos componentes da demanda residencial por energia elétrica. Para isso, o conhecimento das elasticidades fornece um meio de investigar esta relação. Esta pode ser descrita como a variação percentual da quantidade demandada de um bem, em função de uma dada variação percentual de uma variável. O mesmo pode ser representado por:

$$
\eta=\frac{\Delta \% \mathrm{Q}_{\mathrm{i}}(\mathrm{p}, \mathrm{y})}{\Delta \% X_{i}}
$$

Onde:

$\eta$ é a elasticidade;

$\Delta \% \mathrm{Q}_{\mathrm{i}}$ é a variação percentual da quantidade demandada de um bem $\mathrm{i}$; 
$\Delta \% X_{i}$ é a variação percentual de uma variável $X_{i}$ qualquer;

Em outras palavras, a elasticidade sugere qual a intensidade da reação do consumidor sobre a quantidade demandada ante as mudanças na renda e nos preços. Através da estimação da função de demanda e, consequentemente, das elasticidades é possível avaliar como os componentes da demanda se relacionam, como também avaliar a sensibilidade e o impacto de cada uma delas no consumo de eletricidade das famílias brasileiras no período 2004-2015.

\section{Modelo analítico}

Algumas considerações devem ser feitas para utilização do método a ser empregado. A primeira delas é a adoção da função Cobb-Douglas. As características contidas na função simplificam a análise do problema, visto que após a estimação da equação de demanda, os próprios coeficientes de regressão são as elasticidades, além de se manterem constantes ao longo do tempo.

Segundo Andrade e Lobão (1997), algumas hipóteses devem ser adotadas ao modelo para estimar a função de demanda de energia elétrica:

1. Aceita-se, convencionalmente, que a oferta de energia elétrica seja infinitamente elástica e que não exista demanda reprimida por parte dos consumidores atrelados à rede de distribuição de energia elétrica. Ou seja, toda energia elétrica demandada pelas unidades consumidoras deverá efetivamente ser entregue.

2. Considera-se que a demanda por energia elétrica é influenciada ao menos por três variáveis fundamentais: a tarifa residencial cobrada pelo serviço de distribuição, a renda do consumidor e o estoque de equipamentos elétricos, representados algebricamente a seguir:

$$
C_{t}=k P_{t}^{\alpha} Y_{t}^{\beta} E_{t}^{\gamma} \varepsilon^{\mu}, \operatorname{com} k>0, \alpha<0, \beta>0, \gamma>0
$$

Onde:

$k$ é uma constante;

$C_{t}$ é o consumo residencial de energia elétrica no tempo t;

$P_{t}$ é a tarifa média residencial de energia elétrica no tempo t;

$Y_{t}$ é o rendimento médio do trabalhador no tempo t;

$E_{t}$ é o estoque de equipamentos elétricos;

$\varepsilon_{t}$ é o termo de erro aleatório.

Supõe-se que, coeteris paribus, os consumidores residenciais reagem negativamente a elevação no preço da tarifa residencial, reduzindo o consumo de energia elétrica, enquanto um 
aumento da renda do consumidor aumento e/ou estoque de equipamentos elétricos provocará variações positivas no consumo de eletricidade:

3. O estoque de equipamentos elétricos e dispositivos eletrônicos, que via de regra utilizam energia elétrica em seu funcionamento, sofrem depreciação ao longo do tempo. Dado isso, a reposição ou incremento de outros eletrodomésticos estarão sujeitos à renda do consumidor $Y_{t}$ e ao preço $P E_{t}$ dos eletrodomésticos. Algebricamente, essa condição é expressa por:

$$
E_{t}=k_{2} P E_{t}^{\mu} Y_{t}^{\theta}
$$

Espera-se que, coeteris paribus, um aumento da renda do consumidor provoque variações positivas no estoque domiciliar, enquanto a elevação de preços dos equipamentos elétricos ocasiona impactos negativos na variável dependente.

Substituindo (6) em (5) chega-se a equação para demanda residencial de energia elétrica denotada por (7).

$$
C_{t}=k P_{t}^{\alpha} Y_{t}^{\beta} P E_{t}^{\gamma} \varepsilon_{t}^{\mu}, \operatorname{com} k>0, \alpha<0, \beta>0, \gamma<0
$$

Aplicando o logaritmo natural ${ }^{2}$ a equação (7), obtém-se:

$$
\log C_{t}=\log k+\alpha \log P_{t}+\beta \log Y_{t}+\gamma \log P E_{t}+\mu_{t}
$$

Em que:

$\log k$ é o logaritmo da constante;

$\log C_{t}$ é o logaritmo da razão do consumo residencial de energia elétrica no tempo t;

$\log P_{t}$ é o logaritmo da tarifa média residencial de energia elétrica no tempo t;

$\log Y_{t}$ é o logaritmo do rendimento médio do trabalhador no tempo t;

$\log P E_{t}$ é o logaritmo do preço dos equipamentos elétricos;

$\mu_{t}$ é o termo de erro aleatório.

Os demais parâmetros são: $\alpha$ é a elasticidade-preço da tarifa, $\beta$ é a elasticidade-renda, $\gamma$ é a elasticidade-preço dos eletrodomésticos e k é uma constante.

Segundo a teoria econômica, espera-se que o logaritmo do intercepto e da renda se relacione positivamente com o consumo. Em contrapartida, o resultado esperado para o logaritmo dos preços da tarifa e dos equipamentos de eletrodomésticos sejam negativos.

Para a estimação, optou-se pela utilização de séries temporais, visto que dessa maneira dispõe-se de observações agregadas de forma ordenada ao longo do tempo, o que permite a análise de componentes embutidos ou aleatórios, diferentemente de uma relação de causa e efeito expressas em um modelo de regressão simples.

\footnotetext{
2 Os parâmetros se transformam nas próprias elasticidades ao linearizar o modelo através da aplicação do logaritmo na função de demanda.
} 
Nesse sentido, os modelos de séries temporais se baseiam na noção de que uma série $Y_{t}$ é gerada a partir de um processo estocástico, ou seja, processos onde as características probabilísticas são constantes ao longo do tempo. Quando estas não são estacionárias, é necessário utilizar o modelo em diferença da série em questão. Contudo, este procedimento desconsidera as informações de longo prazo, posto que a informações de nível se perdem no momento em que são transformadas em taxas de variação.

No intuito de evitar resultados espúrios, Schmidt e Lima (2005) sugerem a estimação das elasticidades da demanda Residencial por dois métodos: equações simultâneas ou pelo método de cointegração, com a utilização do modelo de vetores autorregressivos (VAR).

Desta maneira, optou-se pelo VAR neste trabalho, visto que este tipo de modelagem possui características essenciais para estimação dos dados. A preferência se deu pelo controle da simultaneidade das variáveis envolvidas neste processo e pela captação das tendências de longo prazo das variáveis.

Godfrey para identificar se o modelo possui ou não autocorrelação serial.

Quadro 1- Descrições e fontes dos dados utilizados na estimação da demanda residencial

\begin{tabular}{|c|l|l|}
\hline \multicolumn{3}{|c|}{ DEMANDA RESIDENCIAL POR ENERGIA ELÉTRICA (2004 - 2015) } \\
\hline C & Consumo Total da Classe Residencial (MWh) & Empresa de Pesquisa Energética (EPE). \\
\hline Y & $\begin{array}{l}\text { Rendimento Médio Nominal Efetivo (R\$) } \\
\text { Valores Constantes: Dezembro de 2015 }\end{array}$ & $\begin{array}{l}\text { IBGE - Pesquisa Mensal do Emprego } \\
\text { (PME). }\end{array}$ \\
\hline P & $\begin{array}{l}\text { Tarifa Média Residencial (R\$MWh) } \\
\text { Valores Constantes: Dezembro de 2015 } \\
\text { Base: Dezembro 2015 }\end{array}$ \\
\hline PE & $\begin{array}{l}\text { Índice de Preços Amplos - OG - Máquinas, Aparelhos } \\
\text { e Materiais Elétricos } \\
\text { Base: Dezembro de 2015 = 100 }\end{array}$ & $\begin{array}{l}\text { Agência Nacional de Energia Elétrica } \\
\text { (ANEEL). } \\
\text { Deflator IGP-DI } \\
\text { Base: Dezembro 2015 }\end{array}$ \\
(IBRE/FGV). \\
\hline
\end{tabular}

Fonte: Elaborado pelos autores.

Adotado o modelo, é preciso considerar a influência da sazonalidade nas variáveis, já que as séries temporais utilizadas neste trabalho são de periodicidade mensal. O componente sazonal pode induzir a conclusões errôneas sobre as séries, sendo necessário seu ajuste. Para tanto, realizou-se o ajuste sazonal pelo programa X12-ARIMA ${ }^{3}$.

${ }^{3}$ O método X12-ARIMA, desenvolvido pelo U.S Census Bureal, foi realizado no software Eviews 7.0. 
Em seguida, se faz necessário verificar se as séries em questão são estacionárias em nível, já que só é possível a utilização do método de cointegração caso haja indícios que a série seja não-estacionária e possua a mesma ordem de integração. Para isso, foi utilizado do teste de raiz unitária por meio do método Argumented Dickey-Fuller ${ }^{4}$ - (ADF).

Ainda, é importante identificar se os resíduos da regressão são autocorrelacionados, uma vez que a correlação serial pode afetar a variância dos parâmetros, invalidando os testes de hipóteses e viesando os estimadores. Dessa maneira, será realizado o teste Breusch-

Além disso, para garantir a condição de estabilidade da estrutura do modelo ao longo do tempo, será feito o teste de Chow. Neste teste, a amostra é subdividida em duas partes e, por meio do teste $\mathrm{F}$, é possível detectar a presença de quebra estrutural.

Posteriormente, o teste de cointegração será realizado para determinar se as séries temporais envolvidas possuem ou não uma relação de longo prazo. Cabe ressaltar que a escolha pelo VAR e emprego do teste cointegração de Johansen $(1988,1991)$ e ainda Johansen e Jucelius (1990) têm sido amplamente utilizados nos trabalhos desenvolvidos sobre demanda residencial de energia elétrica em âmbito nacional e internacional.

A princípio, os dados para estimação da demanda residencial por energia elétrica poderiam ter três periodicidades: mensal, trimestral e anual. Em função da disponibilidade de muitals observações em um curto período de tempo, foi escolhida a periodicidade mensal. Para tanto, o trabalho conta com dados compreendidos o período de janeiro de 2004 a dezembro de 2015. Este período foi escolhido por não apresentar sinais de demanda reprimida e tampouco haver mudanças estruturais significativas no SEB.

No Quadro 1, são apresentadas as descrições e fontes das informações utilizadas no trabalho.

\section{Análise descritiva}

De acordo com a EPE (2016), a classe residencial representava aproximadamente $24 \%$ do consumo total de eletricidade ao final de 2004, o equivalente ao consumo anual de 78.470 gigawatts-hora. Transcorridos onze anos, pôde-se observar que o consumo desta classe cresceu substancialmente, registrando 131.314 gigawatts-hora, aumentando a sua representatividade para $28 \%$ no consumo total. Logo, verificou-se uma expansão de 4,6\% entre os anos de 2004 a 2015.

\footnotetext{
${ }^{4} \mathrm{O}$ teste Argumented Dickey-Fuller (ADF) foi realizado no software Eviews 7.0.
} 


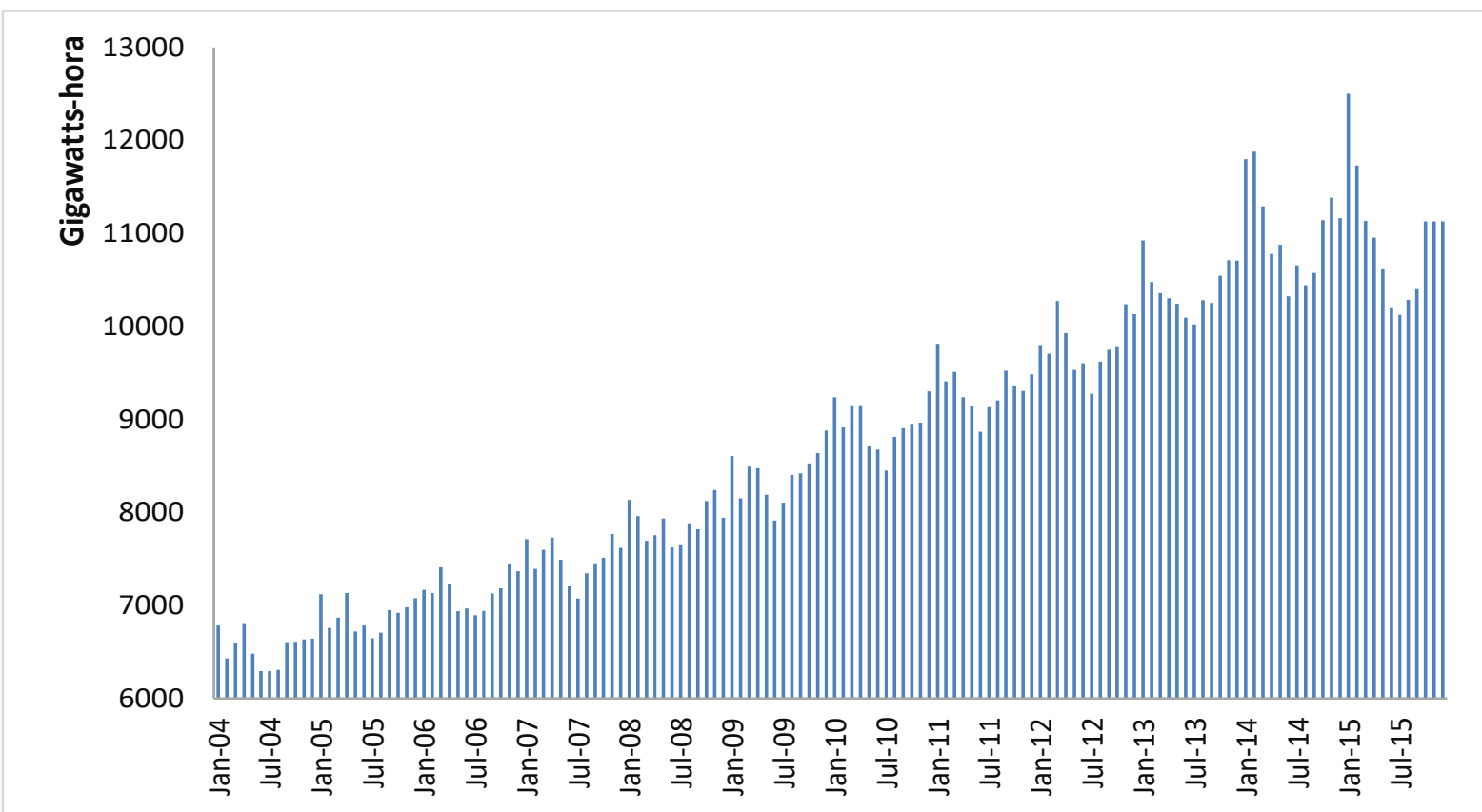

Figura 1 - Evolução do consumo de energia elétrica no Brasil entre 2004-2015

A Figura 1, apresenta a evolução do consumo de eletricidade nas residências brasileiras entre janeiro de 2004 até dezembro de 2015.

Já a tarifa de energia elétrica se manteve no mesmo patamar de janeiro de 2004 até o final de 2012. Em 2013, com a prorrogação das concessões ${ }^{5}$, o governo propôs uma queda das tarifas de energia elétrica praticadas pelas distribuidoras da ordem de $20 \%$.

Entretanto, se tornou insustentável a manutenção dos preços tarifários, visto que em meados de 2013 o volume de água nos reservatórios começou a reduzir consideravelmente, devido ao baixo índice pluviosidade neste período. Este episódio levou o governo a acionar as termelétricas, ação que encareceu o preço da energia elétrica no país.

Em 2015, é notória a mudança de patamar no preço da tarifa média cobrada pelo serviço de energia elétrica. Essa elevação foi consequência do realinhamento dos preços administrados, em especial o de energia elétrica, que estava defasado desde 2013. O reajuste das tarifas, juntamente com a revisão tarifária extraordinária (RTE), parecem ter afetado consideravelmente os preços de energia elétrica neste ano. Abaixo, é possível observar na Figura 2 a evolução das tarifas médias entre janeiro de 2004 até dezembro de 2015.

O rendimento médio real efetivo - medida que leva em consideração o rendimento bruto do trabalho, deflacionado pelo IGP-DI em valores constantes de 2015 - apresenta crescimento em todo o período compreendido entre janeiro de 2004 até o final de 2015 . A estabilidade da

\footnotetext{
${ }^{5}$ São os contratos de concessão assinados entre a Agência Nacional de Energia Elétrica e as empresas prestadoras dos serviços de transmissão e distribuição de energia. (ANEEL, 2010).
} 
economia brasileira, a distribuição da renda mais equitativa e o bom desempenho das exportações líquidas no período, consequência da elevação dos preços das commodities, foram os principais fatores que produziram o crescimento constante, representado por uma taxa média de crescimento da ordem de $2,6 \%$ nos últimos onze anos.

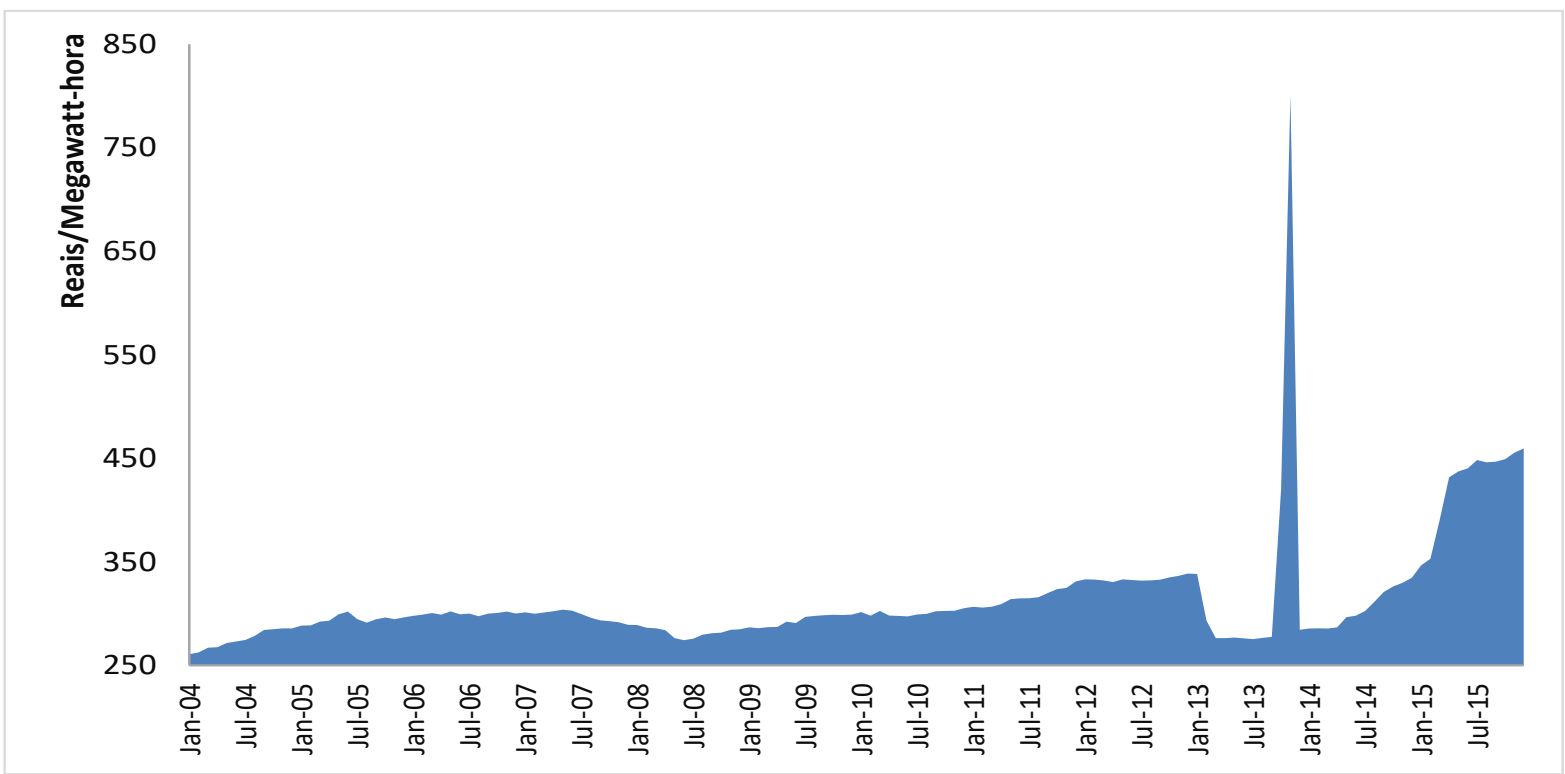

Figura 2 - Evolução da tarifa residencial de energia elétrica no Brasil entre 2004-2015

No entanto, a renda real começou a decrescer em 2015, consequência da inflação causada pelo realinhamento dos preços administrados, juntamente com a recessão do produto interno, reduzindo assim, o poder de compra dos indivíduos. Na Figura 3, é perceptível tais movimentos no rendimento médio real efetivo.

\section{Elasticidade renda da demanda residencial por energia elétrica}

Antes de realizar os testes, especificar o modelo e analisar a cointegração das séries, é necessário analisar se existem componentes sazonais. Em geral, séries de periodicidade mensal possuem sazonalidade e, para eliminar este efeito indesejável nas variáveis do modelo, foi realizado o ajuste sazonal pelo método X12-ARIMA.

Após o ajuste, o logaritmo natural foi aplicado em todas as variáveis para linearizar o modelo e também para evitar problemas com a heterocedasticidade. A utilização das variáveis na forma de logaritmo também se justifica pelo fato de que as estimativas dos coeficientes serem as próprias elasticidades. 


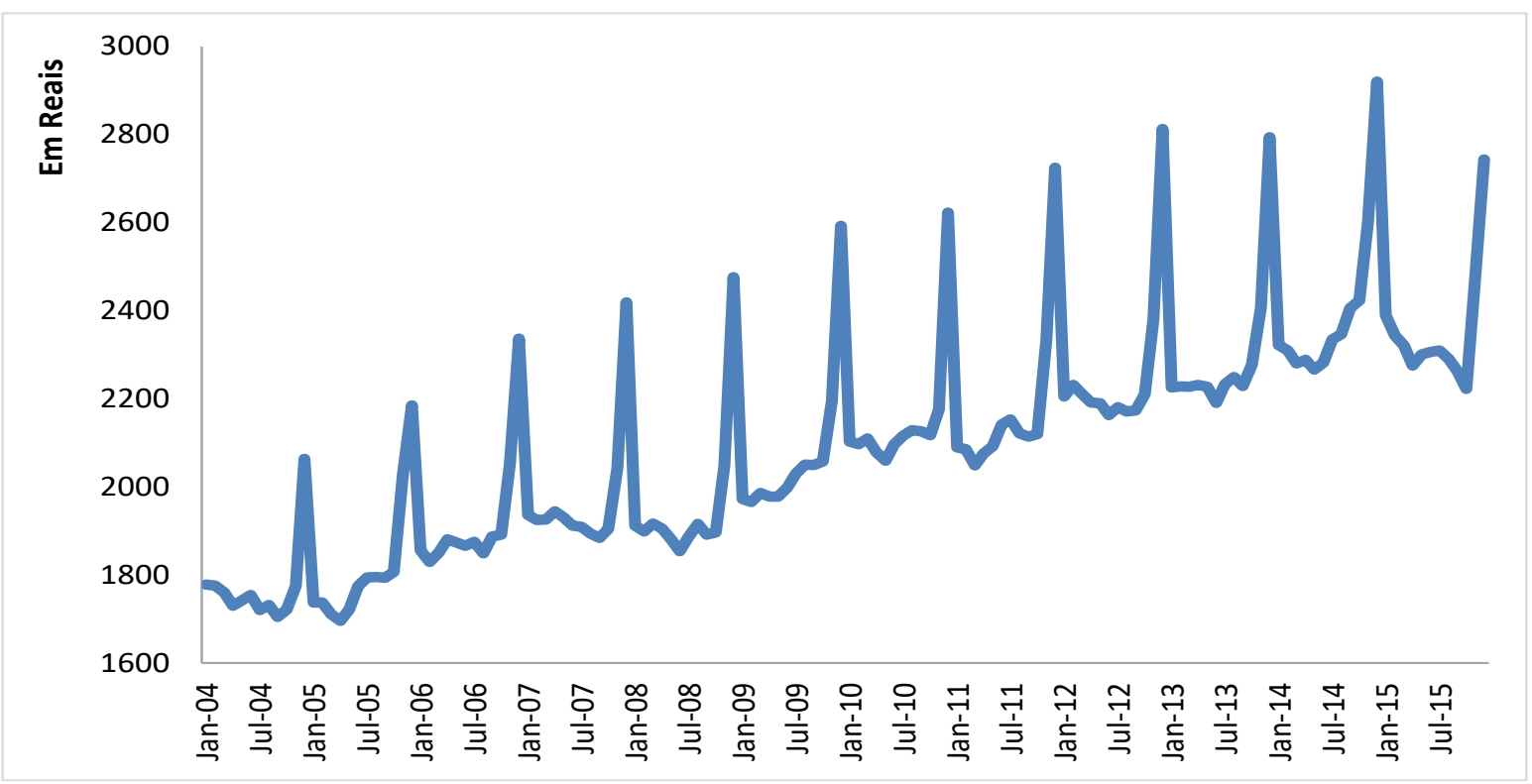

Figura 3- Evolução do Rendimento Médio Real Efetivo entre 2004-2015

Para que seja possível realizar o teste de cointegração, as séries devem ser estacionárias e possuírem a mesma ordem de integração. Neste caso, utilizou-se o teste ADF para verificar a existência de raiz unitária. Os resultados obtidos estão expressos na Tabela 1.

Foram adotadas 13 defasagens para o teste, critério padrão do teste no software Eviews 7. Para a inclusão de constante e tendência em cada uma das séries, foi observado sua evolução e o comportamento apresentado por meio dos gráficos de linha.

Em geral, séries econômicas não são estacionárias em nível, sendo necessária a sua diferenciação. Como pode ser observado na Tabela 1, a um nível de significância de 5\%, nenhuma das variáveis em nível foi capaz de rejeitar a hipótese nula de que a série contém uma raiz unitária, demonstrando o caráter não-estacionário das séries.

Tabela 1 - Teste da raiz unitária

\begin{tabular}{ccccc}
\hline $\begin{array}{c}\text { Variável em } \\
\log \end{array}$ & Termos da Equação & Estatística do & \multicolumn{2}{c}{ Valor Crítico } \\
\hline $\mathrm{C}$ & Coste (ADF) & $1 \%$ & $5 \%$ \\
\hline$\Delta \mathrm{C}$ & Constante e tendência & $-2,59$ & $-4,02$ & $-3,44$ \\
\hline $\mathrm{Y}$ & Constante e tendência & $-13,81$ & $-4,02$ & $-3,44$ \\
\hline$\Delta \mathrm{Y}$ & Constante e tendência & $-2,36$ & $-4,02$ & $-3,44$ \\
\hline $\mathrm{P}$ & Constante & $-16,61$ & $-4,02$ & $-3,44$ \\
\hline$\Delta \mathrm{P}$ & Constante & $-2,19$ & $-3,47$ & $-2,88$ \\
\hline $\mathrm{PE}$ & Constante e tendência & $-11,21$ & $-3,47$ & $-2,88$ \\
\hline$\Delta \mathrm{PE}$ & Constante e tendência & $-9,83$ & $-4,02$ & $-3,44$ \\
\hline $\mathrm{F}$ & & & $-4,02$ & $-3,44$ \\
\hline
\end{tabular}

Fonte: Elaborado pelos autores. 
Desta maneira, foi necessária a realização do teste em primeira diferença e logo verificouse que, ao nível de $1 \%$ de significância, todas as séries rejeitaram a hipótese nula.

Portanto, torna-se evidente que as séries econômicas utilizadas são $I(1)$, ou seja, elas são estacionárias e integradas de ordem (1).

Tabela 2 - Teste de autocorrelação serial

\begin{tabular}{ccc}
\hline Defasagens & Estatística do teste & P-valor \\
\hline 1 & 8,45 & 0,93 \\
\hline 2 & 19,18 & 0,26 \\
\hline 3 & 4,99 & 0,99 \\
\hline 4 & 19,56 & 0,24 \\
\hline 5 & 16,40 & 0,43 \\
\hline 6 & 12,70 & 0,69 \\
\hline 7 & 12,60 & 0,70 \\
\hline 8 & 15,89 & 0,46 \\
\hline 9 & 15,31 & 0,50 \\
\hline 10 & 11,40 & 0,78 \\
\hline 11 & 7,98 & 0,95 \\
\hline 12 & 16,65 & 0,41 \\
\hline
\end{tabular}

Fonte: Resultados da pesquisa.

Para encontrar a ordem de defasagem, buscou-se estimar o modelo com a maior ordem possível e que simultaneamente satisfaça a condição de estabilidade do VAR. Adicionalmente, considerou-se os valores dos critérios de Akaike, Schwarz e Hanna-Quinn para a melhor especificação do modelo.

Embora a escolha da ordem do VAR seja arbitrária, é desejável incluir o maior número possível de defasagens para evitar a imposição de restrições falsas sobre a dinâmica do modelo e garantir que os erros não sejam auto-correlacionados. De acordo com os critérios mencionados acima, o modelo se mostra melhor especificado quando estimado com 12 defasagens, determinando um VAR (12).

Tabela 3 - Teste da raiz unitária para os resíduos em nível

\begin{tabular}{ccccc}
\hline Variável & Termos da & Estatística do teste & \multicolumn{2}{c}{ Valor Crítico } \\
\cline { 4 - 5 } & Equação & (ADF) & $1 \%$ & $5 \%$ \\
\hline $\boldsymbol{\mu}_{\boldsymbol{t}}$ & Nenhum & $-11,60$ & $-2,58$ & $-1,94$
\end{tabular}

Fonte: Resultados da pesquisa.

Especificado a ordem de defasagem do modelo, foi aplicado o teste de Chow para verificar a estabilidade estrutural do VAR (12). O teste indica que as duas partes da amostra possuem a mesma variância, ao aceitar a hipótese nula de que não há quebra estrutural no 
modelo. Logo, o VAR (12) é um bom candidato a ser um modelo bem especificado para a demanda residencial por eletricidade.

No entanto, ainda é necessário analisar se os resíduos contêm auto-correlação serial. A Tabela 2 mostra o teste Breusch-Godfrey.

Ao aceitar a hipótese nula para todos os lags, os resultados do teste apontam a ausência de autocorrelação serial nos resíduos.

Após realizado todo processo de especificação da ordem do modelo, o próximo passo foi verificar a primeira condição para que a serie seja cointegrada. Para isso, é necessário estimar a regressão em nível e verificar se os resíduos são estacionários. A Tabela 3 mostra as estatísticas do teste ADF.

Tabela 4 - Teste de cointegração

\begin{tabular}{ccccccc}
\hline $\begin{array}{c}\text { Número de } \\
\text { vetores }\end{array}$ & $\begin{array}{c}\text { Estatística } \\
\text { do Traço }\end{array}$ & $\begin{array}{c}\text { V. crítico } \\
(5 \%)\end{array}$ & $\begin{array}{c}\text { P-valor } \\
(\%)\end{array}$ & $\begin{array}{c}\text { Est. Máximo } \\
\text { Autovalor }\end{array}$ & $\begin{array}{c}\text { Valor crítico } \\
(5 \%)\end{array}$ & $\begin{array}{c}\text { P-valor } \\
(\%)\end{array}$ \\
\cline { 2 - 7 } Nenhum & 76,92 & 63,88 & 0,00 & 41,42 & 32,12 & 0,00 \\
\hline No máximo 1 & 35,50 & 42,92 & 0,23 & 17,50 & 25,82 & 0,42 \\
\hline No máximo 2 & 18,00 & 25,87 & 0,34 & 12,30 & 19,39 & 0,39 \\
\hline No máximo 3 & 5,69 & 12,52 & 0,50 & 5,69 & 12,52 & 0,50 \\
\hline
\end{tabular}

Fonte: Elaboração dos autores.

Rejeita-se, portanto, a hipótese nula de que os resíduos possuem raiz unitária, o que implica em resíduos estacionários em nível, de ordem de integração $I(0)$.

A segunda condição para a cointegração das séries é a verificação das relações de longo prazo entre as variáveis estimadas e a existência de ao menos um vetor cointegrado para o VAR (12). Para tanto, o teste de cointegração de Johansen (1988) indica se os vetores se cointegram ou não por meio da análise das estatísticas dos testes de Traço e do Máximo Autovalor. Os resultados do teste de cointegração de Johansen é ilustrado na Tabela 4.

De acordo com a Tabelas 4, os testes indicam que, a um nível de significância de 5\%, as variáveis se relacionam no longo prazo e existe ao menos um vetor cointegrado para o modelo. Desse modo, o vetor que determina os valores dos coeficientes e, consequentemente, as elasticidades da equação de demanda residencial por energia elétrica são apresentadas na Tabela 5.

As estimativas da equação (9) sugerem que os coeficientes renda e preço das tarifas são estatisticamente significativos, com sinais esperados de acordo com a teoria econômica.

Portanto, conclui-se que a elasticidade-renda de longo prazo da demanda residencial por energia elétrica é de 0,476 , o que denota um comportamento pouco elástico da renda em relação 
ao consumo. Em termos percentuais, uma variação positiva de $1 \%$ na renda equivale a um impacto positivo de $0,476 \%$ no consumo de energia elétrica.

Tabela 5 - Coeficientes da Demanda Residencial por Energia Elétrica no Brasil

\begin{tabular}{cccccc}
\hline $\boldsymbol{C}$ & $\boldsymbol{K}$ & $\boldsymbol{Y}$ & $\boldsymbol{P}$ & $\boldsymbol{P} \boldsymbol{T}$ & $\boldsymbol{T}$ \\
\hline & $-0,001$ & $-0,476 * * *$ & $0,127^{* * *}$ & $0,276^{* * *}$ & $-0,001 * *$ \\
& & $(0,134)$ & $(0,030)$ & $(0,111)$ & $(0,000)$ \\
\hline
\end{tabular}

Nota: Os valores entre parênteses referem-se ao desvio-padrão.

***: Valor da estimativa a 1\% de significância; ** Valor da estimativa a 5\% de significância;

* Valor da estimativa a $10 \%$ de significância.

Fonte: Resultados da pesquisa.

Logo, a equação da demanda residencial por energia elétrica pode ser expressa por:

$$
C_{t}=0,001+0,476 Y_{t}-0.127 P_{t}-0.276 P_{t}+0,001 T
$$

Quanto à elasticidade-preço de longo prazo da tarifa da demanda residencial, o valor estimado foi de - 0,127, o que representa um comportamento pouco elástico em relação ao consumo. Em termos percentuais, tem-se que uma elevação da tarifa de energia elétrica na ordem de $1 \%$ produzirá uma redução no consumo das famílias em 0,127\%.

Por último, a elasticidade-preço dos equipamentos elétricos foi de - 0,276. Isto significa que uma queda de $1 \%$ no preço dos equipamentos elétricos provocaria um aumento de $0,276 \%$ no consumo de eletricidade.

Painel 1- Estimativas da literatura nacional para a demanda residencial por eletricidade

\begin{tabular}{|c|c|c|c|c|c|c|}
\hline & & Período & Modelo & $\varepsilon_{\mathrm{P}}$ & $\varepsilon_{\mathrm{Pe}}$ & $\varepsilon_{\mathrm{Y}}$ \\
\hline $\begin{array}{c}\text { Modiano } \\
\text { (1986) }\end{array}$ & $\begin{array}{c}\text { Séries } \\
\text { Temporais }\end{array}$ & 1961-1985 & MQO & $-0,403$ & - & 1,13 \\
\hline $\begin{array}{l}\text { Andrade e } \\
\text { Lobão (1997) }\end{array}$ & $\begin{array}{c}\text { Séries } \\
\text { Temporais }\end{array}$ & 1963-1995 & VAR e VEC & $-0,051$ & $-0,186$ & 0,213 \\
\hline $\begin{array}{c}\text { Schmidt e } \\
\text { Lima (2005) }\end{array}$ & $\begin{array}{c}\text { Séries } \\
\text { Temporais }\end{array}$ & 1969-1999 & VAR e VEC & $-0,085$ & $-0,148$ & 0,539 \\
\hline $\begin{array}{l}\text { Gomes } \\
(2010)\end{array}$ & Painel & 1999-2006 & MQO & $-0,089$ & - & 0,091 \\
\hline $\begin{array}{c}\text { Schutze } \\
\text { (2015) }\end{array}$ & Painel & 2005-2014 & MQ2E & $-0,153$ & - & - \\
\hline
\end{tabular}

Fonte: elaborado pelos autores

Observa-se que a estimativa para a elasticidade-renda se aproxima da literatura nacional, segundo os trabalhos apresentados por Andrade e Lobão (1997), Schmidt e Lima (2005) e Gomes (2010). Entretanto, Modiano (1984) apresentam elasticidades-renda superior à este estudo, ao omitir o efeito dos equipamentos elétricos no modelo. Ademais, o resultado obtido 
Revista Catarinense de Economia - RCE

APEC - Associação dos Pesquisadores em Economia Catarinense

$1^{\circ}$ Semestre de 2018 - www.apec.pro.br/rce

para elasticidade-preço é semelhante ao estudo mais recente sobre demanda residencial de energia elétrica, realizado por Schuzte (2015) no período 2005-2014.

Nessa perspectiva a energia elétrica pode ser vista como um bem necessário, na qual uma elevação na renda dos indivíduos provoca o aumento menos do que proporcional na quantidade consumida de energia elétrica nas residências. É válido ainda ressaltar que o crescimento da renda média real dos assalariados nos últimos onze anos provocou alterações positivas no orçamento familiar, o que possibilitou a expansão do consumo de eletricidade e do consumo de outros bens e serviços, aumentando assim o bem-estar dos consumidores brasileiros.

No que diz respeito ao estoque de equipamentos elétricos, reduções em seus preços e aumentos da renda do consumidor provocaram o crescimento da demanda por novos equipamentos, o que, por sua vez, eleva o estoque destes produtos elétricos por domicílio. Estes equipamentos necessitam de eletricidade para executar suas funcionalidades e, por isso, implicam na expansão da quantidade consumida de eletricidade pelos domicílios. À vista disso, a conjuntura econômica brasileira apresentou um quadro de queda real dos preços dos equipamentos elétricos e crescimento real da renda média do assalariado, proporcionando um ambiente ideal para que os brasileiros demandassem mais equipamentos elétricos, o que explica parte da expansão do consumo residencial do Brasil no período.

Em relação à elasticidade-preço, o caráter inelástico da tarifa média cobrada está relacionado com a essencialidade e a falta de bons substitutos para o serviço de eletricidade. Assim, é possível afirmar que aumentos substanciais da tarifa cobrada pelo serviço de energia elétrica não alteram significativamente a quantidade consumida de energia elétrica nas residências. No entanto, o consumidor passará a alocar uma fração maior de sua renda para consumir a mesma quantidade de eletricidade, em detrimento dos outros bens e serviços dos quais são consumidos no dia-a-dia. É o que se tem observado a partir de janeiro de 2015, em que os reajustes elevaram os preços da tarifa média, o que compromete parte do orçamento familiar das famílias brasileiras, gerando uma perda de bem-estar.

\section{Considerações finais}

O presente trabalho teve como objetivo analisar o comportamento da demanda residencial por energia elétrica a nível Brasil, com a finalidade calcular o impacto das elasticidades-renda e dos preços no consumo de energia elétrica entre janeiro de 2004 até dezembro de 2015.

No que tange a modelagem, optou-se pelo VAR por possuir a capacidade de captar relações de interdependência das variáveis, como também de suas respectivas defasagens. Além 
Revista Catarinense de Economia - RCE

APEC - Associação dos Pesquisadores em Economia Catarinense

$1^{\circ}$ Semestre de 2018 - www.apec.pro.br/rce

disso, esta modelagem permite fazer uma análise conjunta com o teste de cointegração. As estimativas encontradas se relacionam no longo prazo, além de serem robustas e estarem acordo com a teoria econômica.

A elasticidade-renda se mostrou menor do que a unidade, indicando que o consumo é pouco sensível à variação na renda do indivíduo. Ademais, a energia elétrica pode ser vista como um bem necessário e, neste caso, um aumento da renda provoca expansão menos do que proporcional ao consumo.

Em relação às elasticidades-preço, observou-se que alterações na tarifa média provocam um impacto menor do que a unidade na retração do consumo de energia elétrica. O resultado inelástico mostra características inerentes ao uso do serviço de energia elétrica, tais como sua essencialidade, como também a falta de um serviço substituto para os lares brasileiros.

Da mesma forma, a elasticidade-preço dos eletrodomésticos também se mostrou inelástica em relação ao consumo. Uma hipótese que se pode levantar é a de que reduções nos preços dos produtos implicam na aquisição de novos equipamentos que, via de regra, necessitam de eletricidade para funcionar, fazendo com que o estoque de produtos eletrodomésticos aumente ao longo do tempo, implicando em um maior consumo de eletricidade nas residências.

Portanto, o conhecimento do tamanho do impacto produzido pela renda, tarifa média e pelo estoque de equipamentos elétricos são necessários para o desenvolvimento de políticas setoriais, em que os órgãos de regulação estabeleçam políticas tarifárias eficientes, visando a melhoria do bem-estar das famílias, em vez de restringir este serviço tão importante nos lares dos brasileiros.

Assim, recomenda-se que seja aprofundada em trabalhos futuros os impactos dos efeitos do realinhamento dos preços administrados praticados a partir de janeiro de 2015, sobretudo para avaliar os efeitos da elevação das tarifas de energia elétrica, já que esta reflete diretamente no comportamento da demanda residencial por energia elétrica.

\section{Referências}

AGENCIA NACIONAL DE ENERGIA ELÉTRICA - ANEEL. Atlas de energia elétrica. Disponível em: < http://www.aneel.gov.br > Acesso em março de 2016.

ANDRADE, T. A.; LOBÃO, W.J.A. Elasticidade renda e preço da demanda residencial de energia elétrica no Brasil. 1997.

BENTZEN, J.; ENGSTED, T. Short-and long-run elasticities in energy demand: a cointegration approach. Energy Economics, v. 15, n. 1, p. 9-16, 1993. 
CORDEIRO JR, H.H; DE SOUSA RAMOS, Francisco Orientador. Previsão de demanda, preço e análise de poder de mercado no setor de energia elétrica. 2005. Tese (Doutorado em Economia) - PIMES, Centro de Ciências Sociais Aplicadas, Universidade Federal do Pernambuco, Recife.

EPE. Ministério de Minas e Energia. Balanço Energético Nacional 2015. Disponível em : < http://www.epe.gov.br > Acesso em outubro de 2015.

EPE. Ministério de Minas e Energia. Balanço Energético Nacional 2016. Disponível em : < http://www.epe.gov.br > Acesso em março de 2016.

GOMES, L. S. F. A demanda por energia elétrica residencial no Brasil: 1999-2006: uma estimativa das elasticidades-preço e renda por meio de painel. 2010. Tese (Doutorado em economia). Universidade de São Paulo, São Paulo.

GUJARATI, D. N.; PORTER, D. C. Econometria Básica. McGraw Hill Brasil, 2011.

HOUTHAKKER, H. S. Some calculations on electricity consumption in Great Britain. Journal of the Royal Statistical Society. Series A (General), v. 114, n. 3, p. 359-371, 1951.

IRFFI, G. et al. Previsão da demanda por energia elétrica para classes de consumo na região Nordeste, usando OLS dinâmico e mudança de regime. Economia Aplicada, v. 13, n. 1, p. 6998, 2009.

JOHANSEN, S. Statistical analysis of cointegration vectors. Journal of economic dynamics and control, v. 12, n. 2, p. 231-254, 1988.

JOHANSEN, S. Estimation and hypothesis testing of cointegration vectors in Gaussian vector autoregressive models. Econometrica: Journal of the Econometric Society, p. 1551-1580, 1991.

JOHANSEN, S.; JUSELIUS, K. Maximum likelihood estimation and inference on cointegration with applications to the demand for money. Oxford Bulletin of Economics and statistics, v. 52, n. 2, p. 169-210, 1990.

LOPES, J. G. Modelo de planejamento da operação de sistemas hidrotérmicos de produção de energia elétrica. 2007. Tese (Doutorado em Economia). Universidade de São Paulo, São Paulo.

MATTOS, L. B.; LIMA, J.E. Demanda residencial de energia elétrica em Minas Gerais: 19702002. Nova Economia, v. 15, n. 3, p. 31-52, 2005.

MODIANO, Eduardo. Elasticidade-renda e preços da demanda de energia elétrica no Brasil. Texto para discussão, 1984.

MONTEIRO, S. Energia Tarifa. Revista Conjuntura Econômica, v. 69, n. 5, p. 50.

OLIVEIRA, A.; SILVEIRA, G. B.; BRAGA, J. M. Diversidade sazonal do consumo de energia elétrica no Brasil. 2000.

OPERADOR DO SISTEMA NACIONAL - ONS. Institucional. Disponível em: < http://www.ons.org.br > Acesso em outubro de 2015. 
PIRES, J. C. L.; GIAMBIAGI, F.; SALES, A. F. As perspectivas do setor elétrico após o racionamento. BNDES, Área de Planejamento, Gerência Executiva de Análise EconômicaGEANE, 2002.

SCHMIDT, C.A.J.; LIMA, M.A.M. A demanda por energia elétrica no Brasil. Revista Brasileira de Economia, v. 58, n. 1, p. 68-98, 2005.

SIQUEIRA, M. L; CORDEIRO JR, H. H.; CASTELAR, I. A demanda por energia elétrica no Nordeste brasileiro após o racionamento de 2001-2002: previsões de longo prazo. Pesquisa e planejamento econômico, v. 36, n. 1, p. 137-178, 2006.

SILK, J. I.; JOUTZ, F. L. Short and long-run elasticities in US residential electricity demand: a co-integration approach. Energy Economics, v. 19, n. 4, p. 493-513, 1997.

SILVA, B. G. Evolução do setor elétrico brasileiro no contexto econômico nacional: uma análise histórica e econométrica de longo prazo. 2011. Tese (Doutorado em economia). Universidade de São Paulo, São Paulo.

SILVA, S. M. Análise econométrica da demanda de energia elétrica nos setores Residencial-Urbano e Rural do Brasil, 1970/1999. 2001. Tese (Doutorado em economia). Universidade Federal de Viçosa, Viçosa.

SCHUTZE, A. M. A Demanda de Energia Elétrica no Brasil. 2015. T Tese (Doutorado em economia). ese de Doutorado. Pontifícia Universidade Católica, Rio de Janeiro.

SONG, H; WITT, S. F. (Ed.). Tourism demand modelling and forecasting. Routledge, 2012.

TOLMASQUIM, M. T. Perspectivas e planejamento do setor energético no Brasil. Estudos Avançados, v. 26, n. 74, p. 247-260, 2012.

VARIAN, H. R. Microeconomia-princípios básicos. Elsevier Brasil, 2006.

VIANA, G. M. N.; SILVA, A.L.M. Um modelo para projeções para demanda por energia elétrica, 2009-2017 para o setor residencial no brasil. 